\title{
Spectral-method algorithm for modeling dispersion of acoustic modes in elastic cylindrical structures
}

\author{
Florian Karpfinger ${ }^{1}$, Henri-Pierre Valero ${ }^{2}$, Boris Gurevich ${ }^{3}$, Andrey Bakulin ${ }^{4}$, and Bikash Sinha ${ }^{2}$
}

\begin{abstract}
A new spectral-method algorithm can be used to study wave propagation in cylindrically layered fluid and elastic structures. The cylindrical structure is discretized with Chebyshev points in the radial direction, whereas differentiation matrices are used to approximate the differential operators. We express the problem of determining modal dispersions as a generalized eigenvalue problem that can be solved readily for all eigenvalues corresponding to various axial wavenumbers. Modal dispersions of guided modes can then be expressed in terms of axial wavenumbers as a function of frequency. The associated eigenvectors are related to the displacement potentials that can be used to calcu-
\end{abstract}

late radial distributions of modal amplitudes as well as stress components at a given frequency. The workflow includes input parameters and the construction of differentiation matrices and boundary conditions that yield the generalized eigenvalue problem. Results from this algorithm for a fluid-filled borehole surrounded by an elastic formation agree very well with those from a root-finding search routine. Computational efficiency of the algorithm has been demonstrated on a four-layer completion model used in a hydrocarbon-producing well. Even though the algorithm is numerically unstable at very low frequencies, it produces reliable and accurate results for multilayered cylindrical structures at moderate frequencies that are of interest in estimating formation properties using modal dispersions.

\section{INTRODUCTION}

Modeling various wave modes propagating along a cylindrical borehole is helpful to understand and allow quantitative interpretation of borehole sonic and seismic measurements. Various modes and head waves propagate in a fluid-filled borehole (Sinha and Zeroug, 1997). Most notably, this includes compressional and shear head waves in addition to the flexural, Stoneley, pseudo-Rayleigh, and leaky modes. Recognizing these head waves and modes further enhances sonic-log quality and validates the use of dispersion curves in related applications. To fully understand and analyze the various modes/head waves present in recorded waveforms, it is of interest to be able to model their characteristics properly as a function of formation, fluid parameters, and geometry.

The classic way to solve such problems is to use a root-finding technique to solve the frequency equation. Although this is not a sig- nificant problem for simple structures such as solid cylinders (Gazis, 1959a, 1959b; Zemanek, 1972), it becomes more complicated for multilayered structures because the separation of the different roots in the complex plane can be a challenging task.

An alternative approach to modeling mode dispersion is to use spectral collocation methods, which are efficient and accurate tools for solving partial differential equations. These methods have been widely used in numerical fluid dynamics (Canuto et al., 1988) as well as in geophysical modeling (Fornberg, 1987; Kosloff et al., 1990; Carcione et al., 2002).

Adamou and Craster (2004) introduce an algorithm based on a spectral Chebyshev scheme that computes the dispersion of circumferential waves in an elastic annulus. Based on this work, Karpfinger et al. (2008a) have developed an algorithm for axisymmetric modes propagating in cylindrical structures with an arbitrary number of fluid and solid layers. This algorithm efficiently computes dispersion

Peer-reviewed code related to this article can be found at http://software.seg.org/2010/0003.

Manuscript received by the Editor 30 January 2009; revised manuscript received 13 August 2009; published online 11 June 2010.

${ }^{1}$ Formerly Curtin University of Technology, Department of Exploration Geophysics, Perth, Western Australia, Australia; presently Schlumberger-Doll Research, Boston, Massachusetts, U.S.A.E-mail: fkarpfinger@slb.com.

${ }^{2}$ Formerly Schlumberger-Doll Research, Boston, Massachusetts, U.S.A.; presently Schlumberger K. K., Japan. E-mail: hvalero@ slb.com; sinha1 @ slb.com; bsinha@ridgefield.oilfield.slb.com.

${ }^{3}$ Curtin University of Technology, Department of Exploration Geophysics, Perth, Western Australia, Australia and CSIRO Petroleum, Perth, Western Australia, Australia.E-mail: b.gurevich@curtin.edu.au.

${ }^{4}$ WesternGeco, Houston, Texas, U.S.A.E-mail: abakulin@ houston.westerngeco.slb.com.

(C) 2010 Society of Exploration Geophysicists. All rights reserved. 
and radial profiles of various modes propagating in such structures.

The spectral method, which we introduce in this article, discretizes the underlying equations with Chebyshev collocation points and differentiation matrices leading to a set of linear equations. We then solve the corresponding system of linear equations as a generalized eigenvalue problem. Note that the eigenvalues we obtain for a given frequency correspond to the slownesses of the different modes.

The purpose of this article is to describe the algorithm and its MATLAB implementation to compute propagating modes in cylindrical structures with arbitrary solid and fluid layers. We briefly discuss the underlying equations and the basic principles of spectral collocation methods. Next, we detail the workflow for implementing the MATLAB code. Following this, we discuss the solution of the generalized eigenvalue problem, which yields eigenvalues and eigenvectors. In addition, we show how we separate the eigenvalues associated with the propagating modes from the nonpropagating modes and spurious eigenvalues. Subsequently, we illustrate how related dispersion curves are computed. Furthermore, we show how eigenvectors, which correspond to the displacement potentials, allow one to compute the distribution of the stress and displacement components along the radius. Finally, we illustrate the results of the spectral-method algorithm using examples from borehole acoustics involving two and four layers.

\section{THEORY}

We develop the theory to compute dispersion curves in cylindrical structures with arbitrary fluid and solid layers using the spectral approach in Karpfinger et al. (2008a). The underlying equations used for the numerical scheme are provided in Appendix A. Equations for an elastic solid and a nonviscous fluid are, respectively, presented in equations A-1-A-10.

For each layer of the considered structure, we compute the differential operator of the fluid $\mathfrak{L}_{\mathrm{fl}}$ (equation A-7) or solid layer. The elastic solid matrix consists of two differential operators as a result of the two differential equations: $\mathfrak{L}_{\mathrm{P}}$ (equation A-1) is the P-wave and $\mathfrak{L}_{\mathrm{S}}$ (equation A-2) is the $S$-wave differential operator. These differential operators are combined in a diagonal block matrix $\mathfrak{L}$. In the case of a

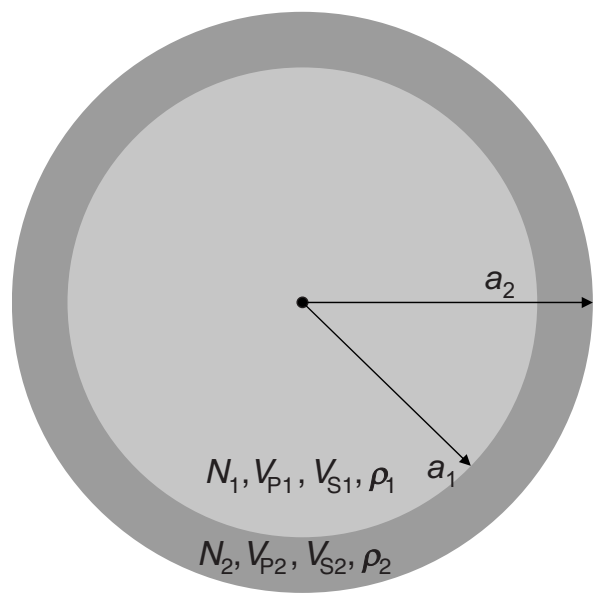

Figure 1. Input parameters for fluid-filled tube - two-layer system. For each layer $i$, the $\mathrm{P}$-wave velocities $V_{\mathrm{P} i}$, $\mathrm{S}$-wave velocities $V_{\mathrm{S} i}$, densities $\rho_{i}$, number of collocation points $N_{i}$, and radii $a_{i}$ must be known. fluid-solid structure (i.e., two-layer system; see Figure 1), the $\mathfrak{L}$ matrix has the form

$$
\mathfrak{L}=\left(\begin{array}{ccc}
\mathfrak{L}_{\mathrm{f} 1} & 0 & 0 \\
0 & \mathfrak{L}_{\mathrm{P}} & 0 \\
0 & 0 & \mathfrak{L}_{\mathrm{S}}
\end{array}\right) .
$$

After discretizing all constitutive equations and introducing related boundary conditions, the problem can be expressed as an algebraic generalized eigenvalue problem:

$$
\widetilde{L} \boldsymbol{\Theta}=k_{z}^{2} \mathbf{M} \Theta
$$

where $\tilde{L}$ is given by equation 1 combined with the stress and displacement components of the boundary conditions. In the diagonal unit matrix $\mathbf{M}$, the stress and displacement components introduced in $\widetilde{L}$ are set to zero. This introduces the boundary conditions into the matrix eigenvalue problem. Solving this eigenvalue problem yields the unknown squared axial wavenumber $k_{z}^{2}$. The axial wavenumber $k_{z}$ is used to compute the phase velocity of the different modes propagating in the structure, and the displacement potential vector $\boldsymbol{\Theta}$ gives the potential for each collocation point along the radial direction and allows computation of stress and displacement components along the radius. For a detailed description of how the eigenvalue problem is formulated and how the $\widetilde{L}$ matrix is constructed, see Karpfinger et al. (2008a).

There are two main differences between the implementation of the spectral method in Karpfinger et al. (2008a) and our implementation in this paper. First, in this paper, we describe fluid layers using the acoustic equation of motion (equations A-7-A-10) rather than the limiting case of the elastic equations. Second, we have discarded the boundary conditions in the center of the structure. Boyd (2001, p. 382-383) shows that no boundary conditions need be set in the center of the structure; for spectral methods, this boundary condition is fulfilled automatically.

\section{SPECTRAL METHOD}

Differential equations can be very efficiently solved with spectral collocation methods. Orthogonal polynomials of high degree are used as global interpolants to approximate the unknown functions of the considered differential equations. The discrete matrix operator, which approximates the differential operator, is called a differentiation matrix. The computation of differentiation matrices can be based on Chebyshev, Fourier, Hermitian, or other interpolants, which can be differentiated exactly. Here, we use the Chebyshev differentiation matrices provided by Weideman and Reddy (2000). The global interpolant evaluated at $N$ interpolation points is connected to its first derivative by a matrix vector product.

Functions interpolated by orthogonal polynomials in evenly spaced points fail to converge for $N \rightarrow \infty$. This is known as the Runge phenomenon; it can be avoided by using unevenly spaced points (Trefethen, 2000). To avoid this effect, Chebyshev points are used to interpolate unknown functions as follows:

$$
x_{j}=\cos \left(\frac{(i-1) \pi}{N-1}\right), \quad j=1, \ldots, N .
$$

The $N$ interpolation points $x_{j}$ are the extrema of the Chebyshev polynomials computed on the interval $[-1,1]$. The collocation points cluster at both ends of the interval. 
Chebyshev points used to discretize an unknown function $f(x)$ interpolated at $N$ nodes $f\left(x_{k}\right)$ can be approximated using interpolant polynomials $\phi_{k}(x)$ :

$$
f(x) \approx \sum_{k=1}^{N+1} f\left(x_{k}\right) \phi_{k}(x) .
$$

Thus, the $\ell$ th derivative of $f(x)$ can be obtained by

$$
f\left(x_{j}\right)^{(\ell)}=\sum_{k=1}^{N+1} \frac{d^{\ell}}{d x^{\ell}}\left(\phi_{k}\left(x_{j}\right)\right) f\left(x_{k}\right) .
$$

If the exact expression of the interpolant polynomial is known, its derivative is obtained exactly. This results in an $N \times N$ differentiation matrix DM:

$$
\mathbf{D M}_{j k}^{(l)}=\frac{d^{l}}{d x^{l}} \phi_{k}\left(x_{j}\right) .
$$

Equation 6 is used to approximate differential operators and therefore can serve to discretize differential equations. Entries of the matrix $\mathbf{D}_{j k}$ and the related code are provided in Trefethen (2000). The $n$th derivative is computed by $n$ matrix multiplications of the first derivative differentiation matrix. The differentiation matrix can be computed with very high spectral accuracy. Nevertheless, round-off errors can be a problem for an increasing number of collocation points. A discussion on how to minimize such errors can be found, for example, in Baltensperger and Trummer (2002).

In the following sections, we show how Chebyshev collocation points and differentiation matrices are used to compute dispersion and radial profiles of waves propagating in cylindrical structures.

\section{STRUCTURE AND ORGANIZATION OF CODE}

Computing dispersion curves in cylindrical structures with arbitrary fluid and solid layers requires various steps, as illustrated in Figure 2. Each step is related to its corresponding MATLAB subroutine:

1) The input parameters of the considered model are set in the DefineModel Params_\#.minput file.

2) Frequency-independent quantities such as differentiation matrices and geometry are subsequently computed in the StructureParameters.m subroutine.

3) Parameters set in previous subroutines are the input of the matrices.m subroutine. This function computes and constructs the matrices for Helmholtz equations $\mathbf{L}$, stress components $\mathbf{S}$, and displacement components $\mathbf{T}$.

4) After these matrices are constructed, the related boundary conditions are set using the eigen.m function. This eigenvalue problem is solved using the standard MATLAB eigenvalue solver eig.

5) Outputs of the computation are the squared eigenvalues and eigenvectors, i.e., $k_{z}^{2}$ and $\theta$ for each frequency.

6) By repeating this workflow (ComputeDispersion.m) for a chosen interval of frequencies, dispersion curves are constructed and plotted with PlotDispersion.m.

7) For a single frequency, eigenvectors are utilized to construct and plot radial profiles (radialprofile.m) as a function of radius structure.
In the following sections, the various steps are described in detail, and we show how they are implemented in the final program.

\section{Input file}

The first step is to set parameters for a certain model. All input parameters are defined in DefineModel Params_\#.m. For each layer, we define the material parameters: $V_{\mathrm{P}}, V_{\mathrm{S}}$, and $\rho$. The geometry of the model is represented by the outer radius $a_{i}$ of each layer (Figure 1). The number of Chebyshev points $N$ for computing differential operators are defined individually for each layer. In addition to these elastic and geometric parameters, we must define the minimum, maximum, and step frequency as wmin, wmax, and ws tep, respectively.

Furthermore, additional parameters must be set. To account for an elastic or a rigid structure, the surface boundary conditions of the structure are set as stress free $s=0$ or rigid $s=1$. Limits for the highest SelectmaxVel = maxvel and lowest Selectminvel = minvel phase velocity can be set to choose a velocity interval of interest.

All of these parameters are entered into a MATLAB structure for code clarity. For a model composed of $j$ layers, the parameter structure has the following form:

Par. $N=[N 1 \ldots N j] ;$ o number of collocation points in each layer

Par. vp $=[\operatorname{vp} 1 \ldots$ vpj] ; $\%$ P-wave velocity of each layer

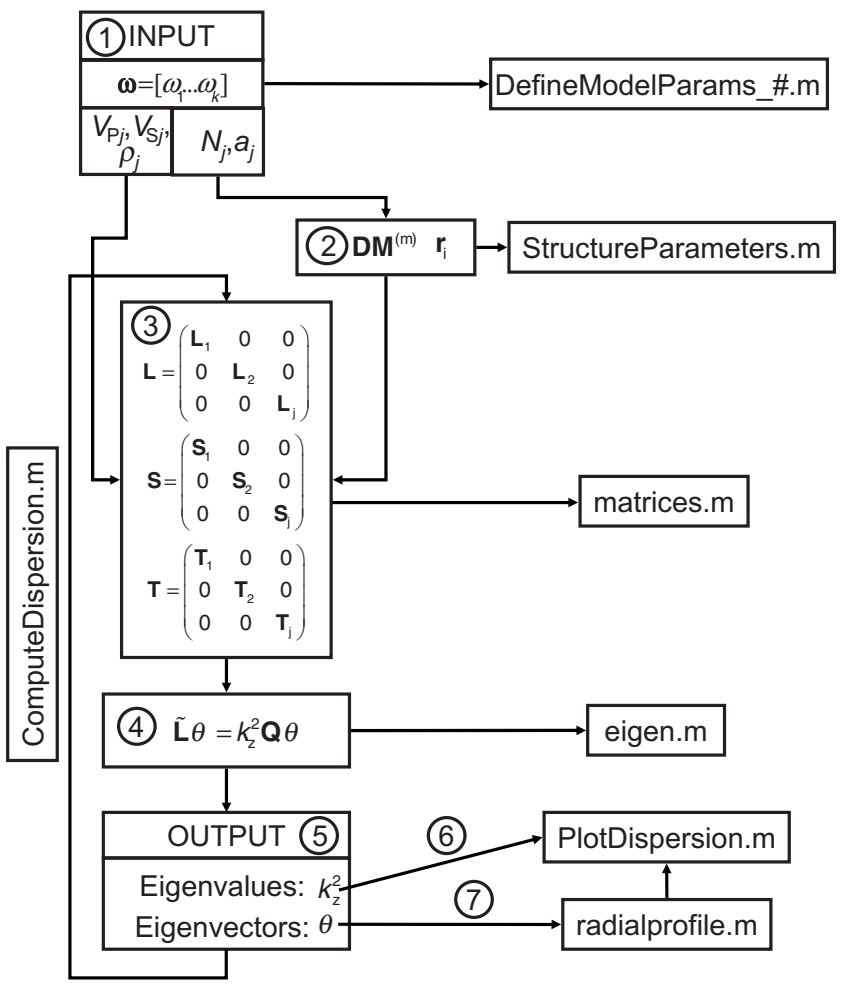

Figure 2. Workflow of the code to compute dispersion and radial profiles. The left side displays how the matrices that define the generalized eigenvalue problem are constructed from the input parameters. Each step is related to its corresponding MATLAB routine on the right side. 


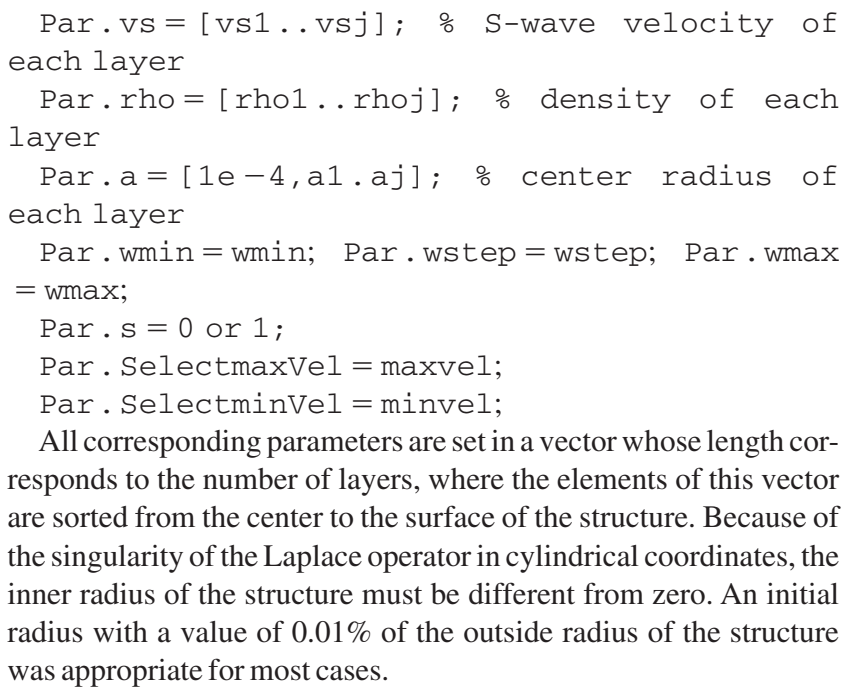

\section{Chebyshev points and differentiation matrices}

The chebdif.m routine produces interpolation points and differentiation matrices for the interval $[-1,1]$. For the case of cylindrical structures, which is considered here, we must transform the interpolation points and differentiation matrices for the minimum and maximum radii of each layer. Each component of the vector Par. $\mathrm{N}_{j}$ corresponds to the number of interpolation points in each layer. All inner and outer radii of the structure are contained in the Par. $a_{j+1}$. Both quantities are used to compute the Chebyshev points and differentiation matrices for each layer of the input model.

We transform the interpolation points $x_{i}$ calculated in equation 3 into radial points $r_{i}$ for each layer $j$ by

$$
r_{i}^{j}=-\frac{\left(a_{j+1}-a_{j}\right) x_{i}+a_{j}+a_{j+1}}{2} .
$$

We do the same for the differentiation matrices produced by the function chebdif, which approximates the $m$ th derivative $d^{m} / d x^{m}$ and can be transformed into a radial dependence $d^{m} / d r^{m}$ as

$$
D_{r}^{m}=-\left(\frac{2}{\text { Par } \cdot \mathrm{a}_{j+1}-\operatorname{Par} \cdot \mathrm{a}_{j}}\right)^{m} D_{x}^{m} .
$$

The radius vector, like the differentiation matrices, is frequency independent and thus computed in SetstructureParameters.m like all other nonfrequency-dependent parameters.

\section{Construction of matrices}

The underlying equations describing axisymmetric wave propagation in solid and fluid media are provided in Appendix A. A detailed derivation of the equation for solids is given in Karpfinger et al. (2008a).

The equations of motion A-1, A-2, and A-7 are ordinary differential equations containing derivatives with respect to $r$ only and coefficients depending on frequency $\omega$ and axial wavenumber $k_{z}$. The aim is to find a relation between $\omega$ and $k_{z}$, which means finding a $k_{z}$ for a given $\omega$, or vice versa. This can be done by solving the equations of motion as an eigenvalue problem so that $k_{z}^{2}$ represents the eigenvalue. Alternatively, we could formulate the problem as a generalized eigenvalue problem in $\omega^{2}$. To do so, we must rearrange equations A-1, A-2, and A-7 so that the terms with $k_{z}$ appear on the left side only. For linear elasticity, both approaches must give identical results. However, for more complicated media (say, viscoelastic or poroelastic), it is advantageous to look for $k_{z}$ as a function of $\omega$ because coefficients of governing equations may themselves explicitly depend on $\omega$.

The wave equations as well as the stress and displacement components, being independent of $k_{z}$ on the left side, are discretized for each layer using Chebyshev interpolation points and differentiation matrices. The matrices of each layer are finally combined in three bigger diagonal block matrices in the matrices . m subroutine.

\section{Helmholtz equation matrix $\mathbf{L}$}

Equation A-7 is the Helmholtz equation for a fluid, whereas equations A-1 and A-2 represent P- and S-wave equations for a solid. The differential operators $\mathfrak{L}_{f \ell}, \mathfrak{L}_{p}$, and $\mathfrak{L}_{s}$ are discretized using the differentiation matrices, the radial collocation points $r_{i}$, and the material parameters. This results in fluid layers in an $N \times N$ matrix $\mathbf{L}_{f \ell}$; for solid layers, $\mathrm{P}$ - and $\mathrm{S}$-wave operators are combined in a $2 \mathrm{~N} \times 2 \mathrm{~N}$ matrix:

$$
\boldsymbol{L}_{s}=\left(\begin{array}{cc}
\boldsymbol{L}_{V \mathrm{P}} & 0 \\
0 & \mathbf{L}_{V \mathrm{~S}}
\end{array}\right)
$$

Finally, all matrices, $\mathbf{L}_{f l}$ and $\mathbf{L}_{s}$, of all layers are combined in one matrix. In the case of a fluid-filled tube, the size of the final matrix $\mathbf{L}$ is $3 N \times 3 N$ and has the form

$$
\mathbf{L}=\left(\begin{array}{cc}
\mathbf{L}_{f \ell} & 0 \\
0 & \mathbf{L}_{s}
\end{array}\right)
$$

\section{Matrices with stress and displacement components}

After we have built the matrix $\mathbf{L}$ representing the differential operators of Helmholtz equations, it is necessary to perform the same operation for the stress and displacement equations (see Appendix A). For a solid layer, the displacement components are equations A-3 and A-4; the stress components are equations A-5 and A-6. The fluid displacement components are equations A-8 and A-9, and the radial stress in the fluid is given by equation A-10. The matrices for the displacement and stress components are constructed in a similar manner to the $\mathbf{L}$ matrix (equation 10 ).

Stress components for a solid layer (defined in Appendix A) can be expressed as

$$
\left(\begin{array}{c}
\sigma_{r r} \\
\hat{\sigma}_{r z}
\end{array}\right)=\underbrace{\left(\begin{array}{cc}
S_{r \Phi} & S_{r \Psi} \\
S_{z \Phi} & S_{z \Psi}
\end{array}\right)}_{S S}\left(\begin{array}{c}
\Phi \\
\hat{\Psi}
\end{array}\right) .
$$

Displacement components (defined in Appendix A) are

$$
\left(\begin{array}{c}
u_{r} \\
\hat{u}_{z}
\end{array}\right)=\underbrace{\left(\begin{array}{cc}
T_{r \Phi} & T_{r \Psi} \\
T_{z \Phi} & T_{z \Psi}
\end{array}\right)}_{T T}\left(\begin{array}{c}
\Phi \\
\hat{\Psi}
\end{array}\right) .
$$

Similarly to the $\mathbf{L}$ matrix, a $3 N \times 3 N$ matrix $\mathbf{S S}$ combining all stress 
coefficients of all layers is constructed:

$$
\mathbf{S S}=\left(\begin{array}{cc}
\mathbf{S}_{f \ell} & 0 \\
0 & \mathbf{S}_{s}
\end{array}\right) .
$$

Likewise, the corresponding displacement coefficient matrix TT can be written as

$$
\mathbf{T} \mathbf{T}=\left(\begin{array}{cc}
\mathbf{T}_{f \ell} & 0 \\
0 & \mathbf{T}_{s}
\end{array}\right) .
$$

SS and TS are $2 N \times 2 N$ matrices (see equations 11 and 12). Each matrix, i.e., $\mathbf{L}, \mathbf{S S}$, and $\mathbf{T T}$, is normalized by the absolute value of its largest element.

\section{Implementing boundary conditions}

While formulating an eigenvalue problem, we must set boundary conditions on the interfaces between layers and on the free surface of the cylinder. The surface and interface boundary conditions are discussed by Karpfinger et al. (2008a). The main emphasis here is to illustrate the implementation of various boundary conditions in the code. The row indices of the matrix $\mathbf{L}$ in which the boundary conditions are introduced are independent of frequency. Thus, we compute these indices in the subroutine SetStructureParameters. $m$. The addition of the boundary conditions into the $\mathbf{L}$ matrix for each individual interface is performed in eigen. $m$. This results in $\mathbf{L}$.

Figure 3 presents the indexing for an $n$-layered system. The interface of interest is between the layers $j$ and $j+1$, which have Par. $N_{j}$ and Par. $N_{j+1}$ interpolation points. In the following equations, we discard Par. for simplicity. To compute the indices of the matrix elements where we want to enter the boundary conditions, we must know the quantity $N A_{j-1}$, the size of the matrix up to the last interface between layers $j-1$ and $j$. It is computed as

$$
N A_{j-1}=\sum_{n=1}^{j-1} N_{n} \mathbf{p},
$$

where $\mathbf{p}$ is a vector of length $j-1$. By convention, components of $\mathbf{p}$ are one if the layer is fluid or two if the layer is solid. Knowing $N A_{j-1}$ for each interface, we can set the boundary conditions for any combination of solid and fluid layers at the interface between the layers $j$ and $j+1$.

For a fluid/fluid interface, the required boundary conditions are the continuity of radial stress and displacement. They are introduced in the following rows:

$$
\begin{gathered}
\left.\sigma_{r r}\right|_{j, j+1}: N A_{j-1}+N_{j}, \\
u_{r \mid j, j+1}: N A_{j-1}+N_{j}+1 .
\end{gathered}
$$

For a fluid/solid interface, in addition to the continuity of radial stress and displacement, the shear stress vanishes in the fluid. The three indices for such an interface are

$$
\left.\sigma_{r r}\right|_{j, j+1}: N A_{j-1}+N_{j}
$$

$$
\begin{gathered}
\left.u_{r}\right|_{j, j+1}: N A_{j-1}+N_{j}+1, \\
\left.\sigma_{r z}\right|_{j, j+1}: N A_{j-1}+N_{j}+N_{j+1}+1 .
\end{gathered}
$$

For a solid/fluid interface, the same interface conditions are considered, but the indices change to

$$
\begin{gathered}
\left.\sigma_{r r}\right|_{j, j+1}: N A_{j-1}+N_{j}, \\
\left.u_{r}\right|_{j, j+1}: N A_{j-1}+2 N_{j}, \\
\left.\sigma_{r z}\right|_{j, j+1}: N A_{j-1}+2 N_{j}+1 .
\end{gathered}
$$

For a solid/solid interface, the following field quantities are continuous: axial and radial displacement as well as normal and shear stress. The indices for such an interface are

$$
\left.\sigma_{r r}\right|_{j, j+1}: N A_{j-1}+N_{j}
$$

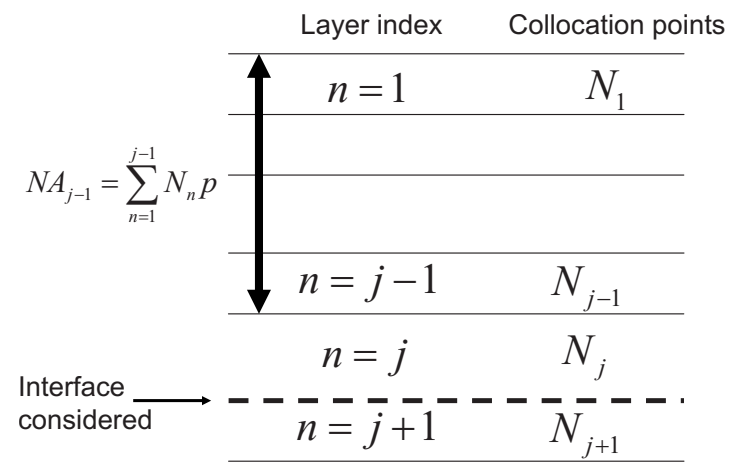

Figure 3. Computation of indices for an interface between layer $n$ $=j$ and $n=j+1$ : to be able to introduce the interface conditions at these layers the size of the matrix from layer $n=1$ up to $n=j-1$ needs to be known: $N A_{j-1}$.

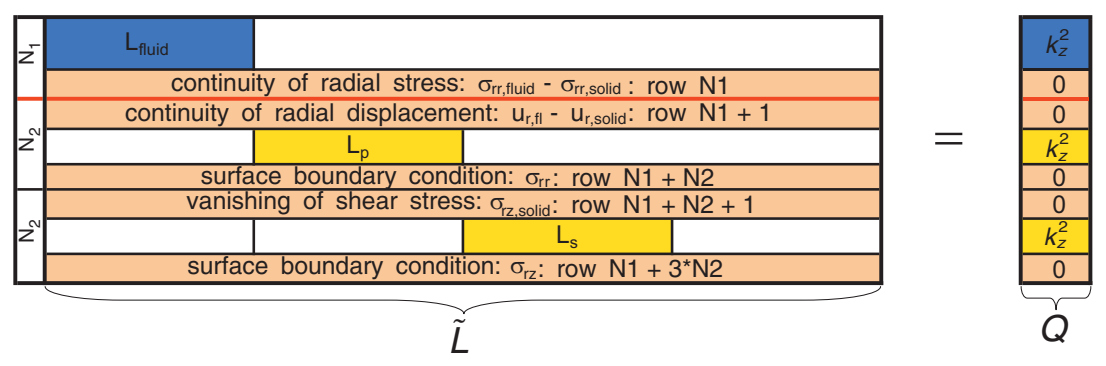

Figure 4. Structure of the matrices $\widetilde{\mathbf{L}}$ and $\mathbf{Q}$ for a borehole surrounded by an elastic formation. The $\widetilde{\mathbf{L}}$ matrix is built of the discretized differential operators of the Helmholtz equations arranged in a diagonal block matrix: fluid layer (blue) and solid layer (yellow); the differential operators of the stress and displacement components corresponding to the boundary conditions for the interface and the surface are set in the orange rows; $\mathbf{Q}$ is a diagonal matrix of the same size as $\mathbf{L}$, where the elements corresponding to the boundary conditions are set equal to zero and the other diagonal elements contain the unknown squared axial wavenumber $k_{z}^{2}$. 


$$
\begin{gathered}
\left.u_{r}\right|_{j, j+1}: N A_{j-1}+2 N_{j}, \\
\left.u_{z}\right|_{j, j+1}: N A_{j-1}+2 N_{j}+1, \\
\left.\sigma_{r z}\right|_{j, j+1}: N A_{j-1}+2 N_{j}+N_{j+1}+1 .
\end{gathered}
$$

Figure 4 illustrates an example of a fluid-solid interface. The interface is indicated with a red horizontal line. The matrix of the fluid (blue) is interpolated by $N_{1}$ points, and the matrix of the solid (yellow) is $2 N_{2} \times 2 N_{2}$. As discussed, three interface conditions are required for this boundary: continuity of radial stress and displacement as well as the vanishing of the shear stress. The boundary conditions are represented by the orange sections. To compute the correct indices for such boundary conditions, we use equations 18-20, where $j=1$ for the fluid and $j=2$ for the solid layer. We can compute the indices of the interface boundary conditions with $N A=0$. Thus, the components of the displacement and stress components are introduced.

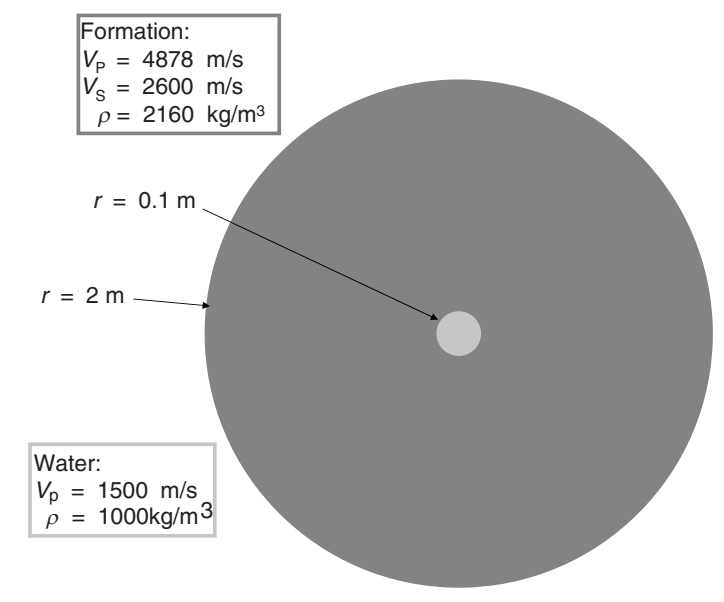

Figure 5. Schematic illustration of a borehole; the borehole is modeled as a finite structure where the outer boundary is 20 times the borehole radius; on the surface, rigid boundary conditions are introduced.

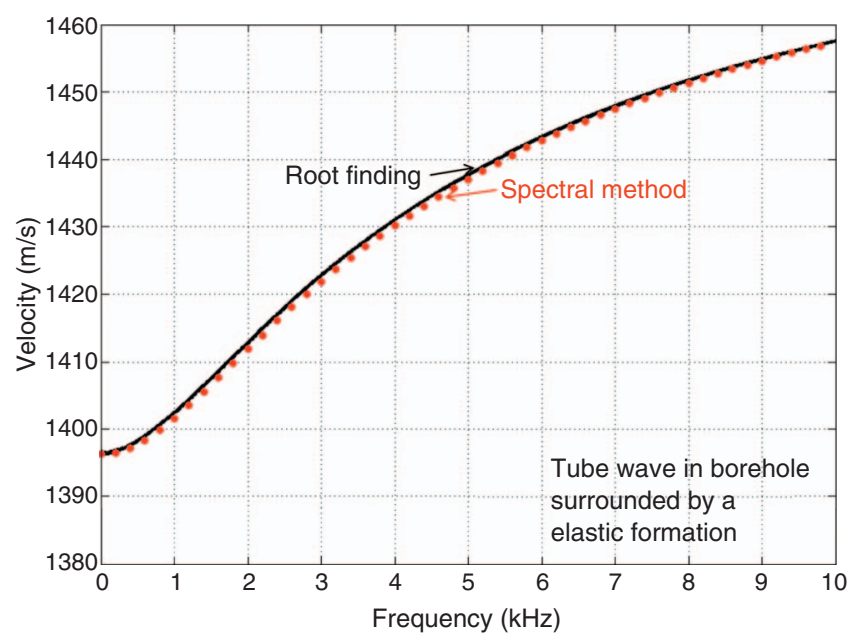

Figure 6. Velocity dispersion of the tube wave computed with the parameters from the model in Figure 5.
To set the boundary condition, we built an identity $\mathbf{M}$ matrix the same size as $\mathbf{L}$. This matrix is normalized with the same value as $\mathbf{L}$ is normalized. In rows where the stress and displacement components are introduced in $\mathbf{L}$, the value of $\mathbf{M}$ is set equal to zero. In a similar manner, we set boundary conditions for all possible interfaces (fluid/ fluid, fluid/solid, solid/fluid, and solid/solid).

After all interface conditions are introduced, it only remains to consider conditions for the surface of the structure. Surface conditions can be selected in the DefineModelParams_\#.m file as stress free $\left(\sigma_{r r}=0\right.$ and $\left.\sigma_{r z}=0\right)$ or rigid $\left(u_{r}=0\right.$ and $\left.u_{z}=0\right)$. These conditions are then set in the rows corresponding to the interpolation points of the surface of the structure. We refer now to the matrix containing all the coefficients for the boundary conditions as $\tilde{\mathbf{L}}$ (see Figure 4).

\section{Eigenvalue problem}

After we have set the boundary conditions, we need to solve a generalized eigenvalue problem of the following form:

$$
\tilde{\mathbf{L}} \boldsymbol{\Theta}=k_{z}^{2} \mathbf{M} \Theta .
$$

We are using the eig eigenvalue solver provided by MATLAB: eig. For each frequency $\omega$, the eigenvalues correspond to the squared axial wavenumber $k_{z}^{2}$. The number of obtained eigenvalues corresponds to the size of the matrix $\tilde{\mathbf{L}}$. It is not straightforward to separate the values of interest from spurious values. The nonpropagating modes, which are not spurious but also not of interest in this study, are excluded by deleting those eigenvalues whose real part is either negative or smaller than the imaginary part. The remaining spurious eigenvalues are easy to distinguish from the desired values because they usually are significantly smaller in the phase velocity domain. Solving the eigenvalue problem for an interval of frequencies gives the dispersion curves of all obtained modes. These curves are constructed and plotted in PlotDispersion.m.

Solving the eigenvalue problem also provides the eigenvectors corresponding to the displacement potentials $\theta$. They can be used to compute the radial distribution of the stress and displacement components along the radius of the structure (step 7, Figure 2). This can easily be achieved by multiplying the potential $\theta$ of each mode with the of the stress and displacement components, SS and TT. The resulting vectors are reshaped in the radialprofile.m subroutine. At the moment, the displacement and stress profiles are computed for an equal number of interpolation points in each layer. The profiles of all different modes for a fixed frequency can be displayed. Radial profiles can help us to better understand the physics of different modes propagating in elastic cylindrical structures.

\section{EXAMPLES}

In our software package, we include two examples. The first is a fluid-filled borehole surrounded by an elastic formation. The medium parameters and the dimensions are given in Figure 5. To compute the dispersion, the parameter file DefineModelParams_1a.m needs to be called in ComputeDispersion.m. The formation is modeled as a finite structure. For an outer radius 20 times bigger than the borehole radius, the tube-wave dispersion is the same as for an unbounded formation.

The result of the eigenvalue problem for a borehole surrounded by an elastic formation is illustrated in Figure 6. The dispersion of the tube wave is computed up to $10 \mathrm{kHz}$ with a frequency step of 
$0.2 \mathrm{kHz}$. We have chosen rigid surface-boundary conditions, so no extensional mode is observed. If we choose the surface-boundary conditions to be stress free, the dispersion of the tube wave will not change. The difference will be that an additional mode, the extensional or Young's modulus mode, will be added and the cutoff frequencies of the higher-order modes will be shifted toward slightly higher frequencies. Because these modes are related to finite structures only, we do not consider this effect in our study.

In Figure 6, we compare the results of the spectral method (red dots) with a dispersion curve obtained from root finding (black line). The root-finding algorithm used to obtain the analytical results is from Sinha and Asvadurov (2004). Both results are in very good agreement. In addition to the tube wave, higher-order modes resulting from reflections on the outer surface of the structure are obtained. These modes are not shown because they are irrelevant for a borehole model. To account for an infinite structure and to avoid the higher-order modes, absorbing boundary conditions will have to be implemented in the future.

For the fluid-filled borehole, the displacement and stress profiles are displayed for $1.5 \mathrm{kHz}$ in Figure 7. The radial distributions of the radial and axial displacement as well as the normal and shear stress along the radius are shown. In this case, the parameter file in ComputeDispersion.m needs to be named DefineModel Params_1b.m. These quantities are plotted as a function of $r / a$, where $r$ is the radius vector and $a$ is the radius of the structure. All radial profiles are normalized by their maximum value. The dashed line indicates the borehole wall. The four plots show that the field quantities are almost zero at a distance of 20 borehole radii. Thus, the tube wave is unaffected by the finite structure.

The second example is a four-layer model shown in Figure 8. Here, ComputeDispersion.m needs to be named DefineModel-Params_2.m. Such geometry is used by Bakulin et al. (2008b) to simulate a well completion in a physical modeling experiment. In our computation, we take the elastic parameters from Bakulin et al. (2008b). The resulting dispersion is displayed in Figure 9. Because of the presence of two fluid columns, two tube waves are supported by this configuration. The fast tube wave is supported mainly by the outer tube; the slow tube wave is supported by the inner tube. In contrast to the first example, we have chosen stress-free surface boundary conditions. This adds two modes that propagate from zero frequency. These modes are called extensional and propagate for low frequencies with the phase velocity of a bar $V_{0}$ $=\sqrt{E / \rho}$, where $E$ is the Young's modulus of each solid layer.

Radial profiles can be computed in the same manner as for the borehole case. In this example, the low-frequency/high-velocity eigenvalues are unstable. Our attempts to overcome this numerical instability have been unsuccessful, and the instability remains to be addressed. We think our present results give reasonable estimates. Because dispersion is very small up to $5 \mathrm{kHz}$, it is possible to extrapolate the results to the correct low-frequency velocity.

For both examples, it is interesting to look at the number of discretization points. In the case of the borehole, the structure is discretized with 10 points in the fluid layer and 40 in the formation. For the four-layer case, the best result can be achieved for eight points in the solid layers and 20 points in the fluid layers. Obviously, more points are needed to model a borehole structure than a four-layer tube. The accuracy of the results can decrease significantly if the number of collocation points is increased. This is especially a problem if some of the layers are very thin, such as the four-layer example. Increasing the number of collocation points for this example by a factor of two or three will make the result more unstable, especially for the higher-order modes. This problem must be considered when implementing new models because the right number of collocation points will most likely require several runs of the same model. In general, for elastic modeling, this is a minor issue because the computing time in most cases is less than $20 \mathrm{~s}$.

\section{DISCUSSION}

In elasticity theory, dispersion equations for guided waves usually are solved for a given wavenumber $k_{z}$ with an unknown frequency $\omega$ (Ewing, 1957; Kolsky, 1963). The same approach can be used in the spectral method, where the equations of motion can be written for $k_{z}$ and the eigenvalues are squared frequencies $\omega$ or velocities $\left(\omega / k_{z}\right)^{2}$. A limitation of this approach is that it cannot be extended to more complex media where coefficients of the constitutive equations explicitly depend on frequency (such as poroelastic media, discussed below). To design an algorithm that might be extended to such media, we have reformulated the elastic problem in such a way to formulate the eigenvalue problem with frequency $\omega$ as a (given) parameter and squared wavenumbers $k_{z}^{2}$ [or slownesses $\left.\left(k_{z} / \omega\right)^{2}\right]$ as eigenvalues.
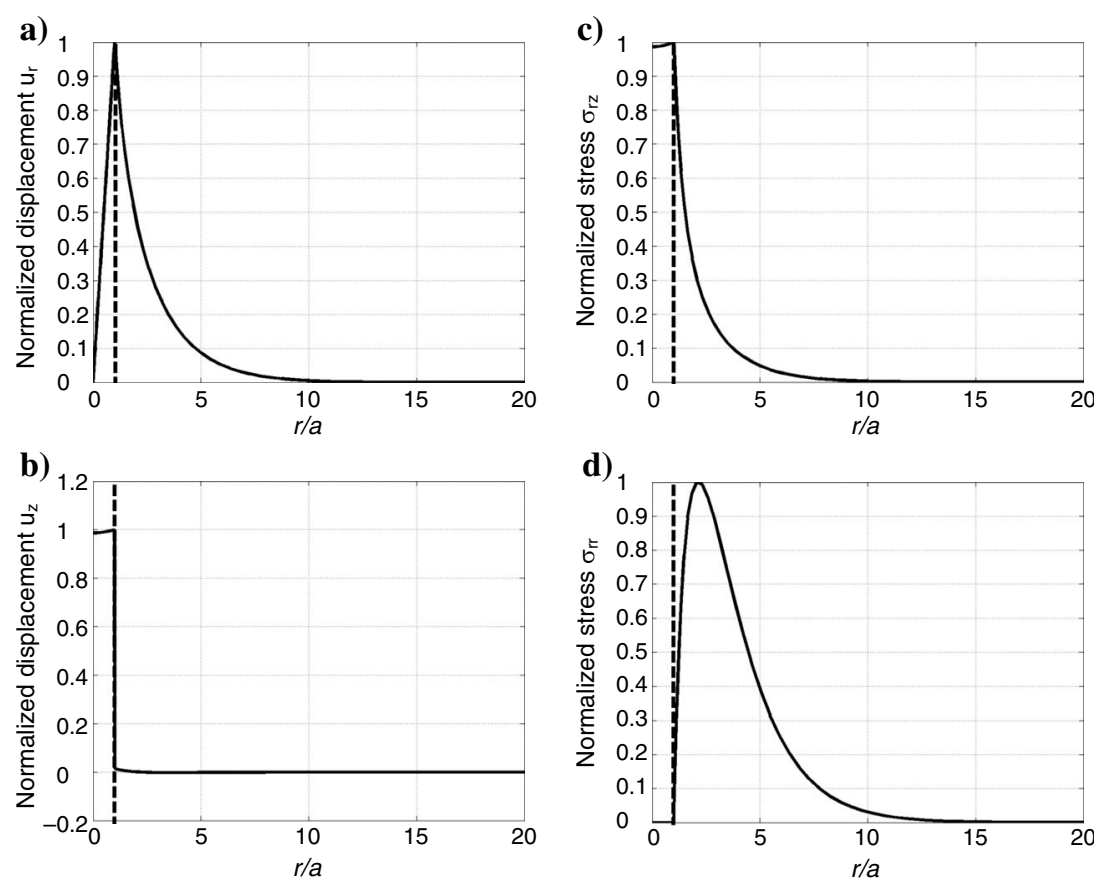

Figure 7. Radial profiles of the tube wave from Figure 6 computed at $1.5 \mathrm{kHz}$; the radial profiles are normalized by their maximum value of the displacements and stresses, respectively; $r / a$ is the radial position $r$ normalized by the radius of the structure $a$. (a) Radial displacement; (b) axial displacement; (c) radial stress; (d) shear stress. 
In an earlier work (Karpfinger et al., 2008a), the fluid is modeled as a limiting case of an elastic solid with a small shear velocity (say, $V_{\mathrm{S}}=1 \mathrm{~m} / \mathrm{s}$ ). This approach results in several additional spurious modes corresponding to fictitious shear waves in the fluid. No difficulties result when the problem is formulated for $k_{z}$ because in this case the eigenvalues (squared velocities) corresponding to the spurious modes are very small and can be ignored. However, in the case of input frequency, the eigenvalues obtained are squared slownesses. For the spurious modes, these slownesses are extremely high. This makes the eigenvalues of interest; they have a much smaller slowness, are numerically unstable and are thus difficult to recover. To obtain results that are more numerically stable, the equations for an ideal fluid (equations A-7-A-10) were implemented in the present algorithm, eliminating these spurious modes altogether. This also resulted in smaller matrices and hence faster computations.

To account for fluid flow across the layer boundaries, we have extended the spectral method to poroelasticity (Karpfinger et al., 2008 b). This allows us to model acoustic response of realistic situations such as a completed borehole surrounded by a formation with a

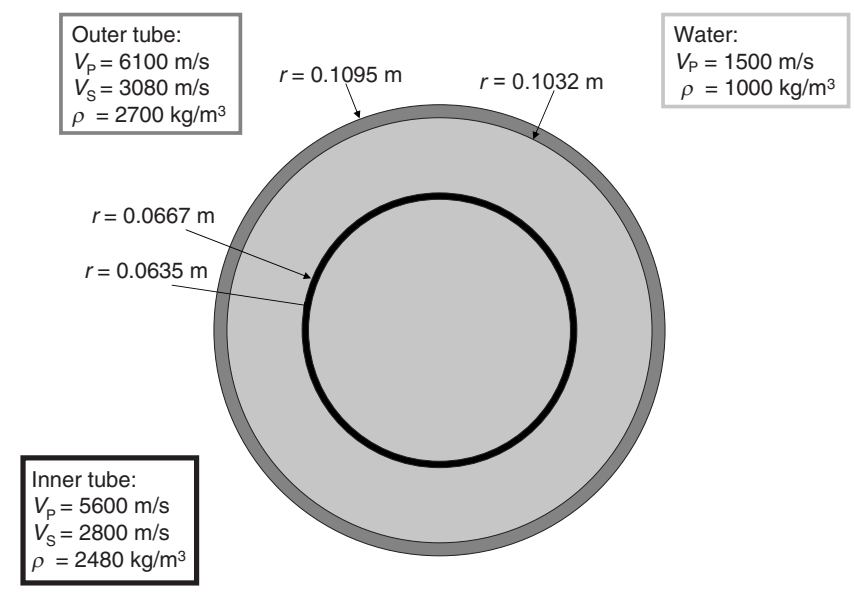

Figure 8. Geometry and elastic parameters of the four-layer model with stress-free surface boundary conditions on the outer layer of the structure.

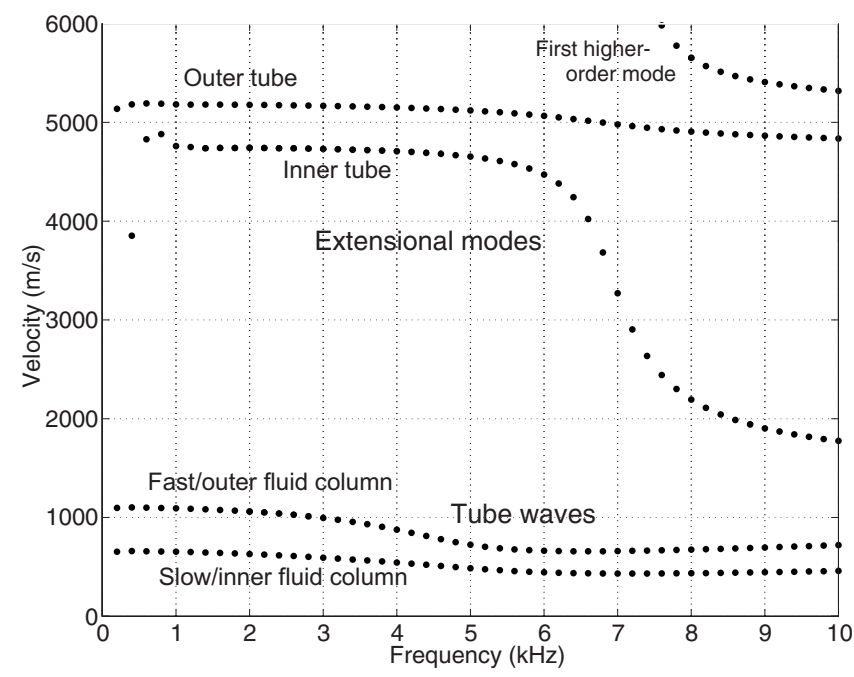

Figure 9. Dispersion of the two extensional modes and two tube waves. radial variation of permeability. Such modeling potentially may be used to monitor reservoir production using permanent acoustic sensors (Bakulin et al., 2008a).

The algorithm presented here is limited to structures of finite radius. Infinite structures, such as a fluid-filled borehole surrounded by an infinite formation, are approximated by a finite structure with a large radius. The down side of this approach is that it produces higher-order modes corresponding to the reflection at the outer boundary of the structure. To avoid these higher-order modes and to be able to model attenuated leaky modes, absorbing boundary conditions must be introduced. This can be the subject of future work. The algorithm also can be extended to flexural waves and anisotropy.

\section{CONCLUSIONS}

We have presented a new algorithm, based on the spectral method, that computes wave propagation in elastic cylindrical structures with an arbitrary number of fluid and solid layers. We discretized the medium in the radial direction using Chebyshev points. We then approximated differential operators by Chebyshev differentiation matrices. The problem was then formulated as an algebraic generalized eigenvalue problem, where the eigenvalues correspond to the axial wavenumbers and the eigenvectors to the displacement potentials. We used the obtained displacement potentials to display radial profiles, which are the variation of displacement and stress along the radius of the structure. Creating an input file for multilayer models is very straightforward, and the results are obtained very efficiently. The algorithm is very fast because of the computational efficiency of the spectral method. It is not straightforward to choose the optimum number of collocation points because there is no parameter to control the accuracy. It will require some runs to get an optimal picture. The two examples indicated how to choose the number of points.

It is not easy to give a general rule; but after running the program for some examples, experience will help users obtain the best possible result. The algorithm works for any number of layers and down to a layer thickness of $0.5 \%$ relative to the thickness of the structure. The drawbacks are that for an increasing number of layers, computing time increases significantly; for very thin layers, accuracy decreases. For most models of interest, the algorithm works very efficiently and is easy to use.

\section{ACKNOWLEDGMENTS}

We are grateful to Boris Kashtan (St. Petersburg State University, Russia), who suggested the idea of applying the spectral method to the problem at hand, and Richard Craster (Imperial College, London) for helpful advice. Florian Karpfinger thanks Shell International Exploration and Production Inc. for support of his $\mathrm{Ph} . \mathrm{D}$. project.

\section{APPENDIX A}

\section{UNDERLYING EQUATION}

\section{Equations for solid media}

The P- and S-wave Helmholtz equations for an isotropic elastic solid expressed in terms of displacement potentials are 


$$
\underbrace{\partial_{r}^{2}+r^{-1} \partial_{r}+\frac{\omega^{2}}{V_{\mathrm{P}}^{2}}}_{\mathfrak{L}_{V_{\mathrm{P}}}}) \Phi=k_{z}^{2} \Phi
$$

and

$$
(\underbrace{\frac{\partial^{2}}{\partial r^{2}}+\frac{1}{r} \frac{\partial}{\partial r}-\frac{1}{r^{2}}+\frac{\omega^{2}}{V_{\mathrm{S}}^{2}}}_{\mathfrak{L}_{V_{\mathrm{S}}}}) \Psi=k_{z}^{2} \Psi .
$$

The radial and axial displacement in such a medium can be expressed as

$$
\begin{gathered}
u_{r}=\partial_{r} \Phi-\hat{\Psi}, \\
\hat{u}_{z}=\underbrace{-k_{z}^{2} \Phi}_{\mathfrak{L}_{V_{\mathrm{P}}} \Phi}+\left(\partial_{r}+r^{-1}\right) \hat{\Psi},
\end{gathered}
$$

where $\hat{\Phi}=i k_{z} \Phi$. The radial normal stress and the axial shear stress are

$$
\begin{gathered}
\sigma_{r r}=\left[2 \mu \partial_{r}^{2}-\lambda \frac{\omega^{2}}{V_{\mathrm{P}}^{2}}+\right] \Phi-2 \mu \partial_{r} \hat{\Psi}, \\
\hat{\sigma}_{r z}=-2 \mu\left(\partial_{r}^{3}+r^{-1} \partial_{r}^{2}-r^{-2} \partial_{r}+\frac{\omega^{2}}{V_{\mathrm{P}}^{2}} \partial_{r}\right) \Phi \\
+\mu\left(2 \partial_{r}^{2}+2 r^{-1} \partial_{r}-2 r^{-2}+\frac{\omega^{2}}{V_{\mathrm{S}}^{2}}\right) \hat{\Psi},
\end{gathered}
$$

where $\hat{\sigma}_{r z}=i k_{z} \sigma_{r z}$.

\section{Equations for fluid media}

The equivalent equations for an ideal fluid are

$$
k_{z}^{2} \Phi=\underbrace{\left(\partial_{r}^{2}+r^{-1} \partial_{r}+\frac{\omega^{2}}{V_{\mathrm{fl}}^{2}}\right)}_{\mathfrak{L}_{V_{\mathrm{fl}}}} \Phi
$$

$$
\begin{gathered}
u_{r}=\partial_{r} \Phi, \\
\hat{u}_{z}=\underbrace{-k_{z}^{2} \Phi}_{\mathfrak{L}_{V_{\mathrm{P}}} \Phi},
\end{gathered}
$$

$$
\sigma_{r r f}=-\lambda \frac{\omega^{2}}{V_{\mathrm{P}}^{2}} \boldsymbol{\Phi} .
$$

\section{REFERENCES}

Adamou, A. T. I., and R. V. Craster, 2004, Spectral methods for modelling guided waves in elastic media: Journal of the Acoustical Society of America, 116, 1524-1535.

Bakulin, A., F. Karpfinger, and B. Gurevich, 2008a, Understanding acoustic response of deepwater completions: The Leading Edge, 27, 260-267.

Bakulin, A., A. Sidorov, B. Kashtan, and M. Jaaskelainen, 2008b, Real-time completion monitoring with acoustic waves: Geophysics, 73, no. 1, E15E33.

Baltensperger, R., and M. R. Trummer, 2002, Spectral differencing with a twist: SIAM Journal on Scientific Computing, 24, 1465-1487.

Boyd, J. P., 2001, Chebyshev and Fourier spectral methods: Dover.

Canuto, C., M. Hussaini, A. Quarteroni, and T. Zang, 1988, Spectral methods in fluid dynamics: Springer Verlag.

Carcione, J. M., G. C. Herman, and A. P. E. ten Kroode, 2002, Seismic modeling: Geophysics, 67, 1304-1325.

Ewing, W. M., 1957, Elastic waves in layered media: McGraw-Hill Book Company, Inc.

Fornberg, B., 1987, The pseudospectral method: Comparisons with finite differences for the elastic wave equation: Geophysics, 52, 483-501.

Gazis, D. C., 1959a, Three-dimensional investigation of the propagation of waves in hollow circular cylinders. I. Analytical foundation: Journal of the Acoustical Society of America, 31, 568-573.

, 1959b, Three-dimensional investigation of the propagation of waves in hollow circular cylinders. II. Numerical results: Journal of the Acoustical Society of America, 31, 573-578.

Karpfinger, F., B. Gurevich, and A. Bakulin, 2008a, Computation of wave propagation along cylindrical structures using the spectral method: Journal of the Acoustical Society of America, 124, 859-865.

- 2008b, Modeling of axisymmetric wave modes in a poroelastic cylinder using spectral method: Journal of the Acoustical Society of America, 124, EL230-EL235.

Kolsky, H., 1963, Stress waves in solids: Dover

Kosloff, D., D. Kessler, A. Q. Filho, E. Tessmer, A. Behle, and R. Strahilevitz, 1990, Solution of the equations of dynamic elasticity by a Chebyshev spectral method: Geophysics, 55, 734-748.

Sinha, B., and S. Asvadurov, 2004, Dispersion and radial depth of investigation of borehole modes: Geophysical Prospecting, 52, 271-286.

Sinha, B., and S. Zeroug, 1997, Geophysical prospecting using sonics and ultrasonics, in J. G. Webster, ed., Wiley encyclopedia of electrical and electronic engineers: Wiley Interscience.

Trefethen, L. N., 2000, Spectral methods in MATLAB: SIAM

Weideman, J. A. C., and S. C. Reddy, 2000, A MATLAB differentiation matrix suite: ACM Transactions on Mathematical Software, 26, 465-519.

Zemanek, J., 1972, An experimental and theoretical investigation of elastic wave propagation in a cylinder: Journal of the Acoustical Society of America, 51, 265-283 